\title{
Reporting Quality Assessment of Randomized Controlled Trials Published in Nephrology Urology Monthly Journal
}

\author{
Alireza Mehrazmay, ${ }^{1}$ Alireza Karambakhsh, ${ }^{1}$ and Mahmood Salesi ${ }^{2,}$ \\ ${ }^{1}$ Behavioral Sciences Research Center, Baqiyatallah University of Medical Sciences, Tehran, IR Iran \\ ${ }^{2}$ Nephrology and Urology Research Center, Baqiyatallah University of Medical Sciences, Tehran, IR Iran \\ "Corresponding author: Mahmood Salesi, Nephrology and Urology Research Center, Baqiyatallah University of Medical Sciences, Tehran, IR Iran. Tel/Fax: +98-9196805469, \\ E-mail:smahmood1360@yahoo.com
}

Received 2015 April 06; Accepted 2015 April 24.

\begin{abstract}
Context: Randomized controlled trials (RCTs) are important tools for evidence-based health care decisions. It is, therefore, important that they be conducted and reported with the highest possible standards. The aim of this study was to evaluate the reporting quality of the RCTs published in nephrology urology monthly journal and to examine whether there was a change over time in the reporting quality.

Evidence Acquisition: The quality of each report was assessed using the Consolidated Standards of Reporting Trials (CONSORT) 2010 Statement checklist and a 5-point quality assessment instrument, i.e. the Jadad scale.

Results: Eighteen (14 Iranian and 4 non-Iranian) RCTs were published from 2012 to 2014 on topics including renal stone (16.6\%), hemodialysis and transplantation (38.8\%), and prostate conditions (11.1\%). Interventions comprised surgery, drugs, and teaching method in 7 (38 \%), 10 (55\%), and 1 (5\%) of them, respectively. According to the CONSORT checklist, the weakest reported items were registration number, identification as a randomized trial in the title, and settings and locations where the data were collected. The mean Jadad score of the reports was $2.72 \pm 1.36$ ( $54 \%$ of their maximum possible total score). According to the Jadad and CONSORT scales, there was an increase in the quality of reporting from 2012 to 2014.

Conclusions: This assessment shows low reporting quality scores in reports. Training courses for researchers, using standard reporting tools (e.g. CONSORT 2010 Statement checklist), and consultation with methodologists can improve the quality of published RCTs.
\end{abstract}

Keywords: Nephrology, Urology, Randomized Controlled Trial, Checklist

\section{Context}

\subsection{Quality Assessment of Randomized Controlled Trials}

In randomized controlled trials (RCTs), the participants are allocated to two groups of intervention and control for the comparison of some outcomes between them (1). RCTs are considered the most valuable method to assess the efficacy of treatments whether or not the result of a comparison is statistically significant. Thus, it is important that they be conducted and reported with the highest possible quality so as to enable the readers to judge which results are internally valid and bias-free. Moreover, it is significant to differentiate between assessing the quality of a trial and the quality of its reporting. The quality of a trial is defined as the confidence that the design, conduct, and analysis of the trial have minimized or avoided biases in its treatment comparisons. This definition focuses on design quality. The quality of a report can be defined as the provision of information about the design, conduct, and analysis of the trial. A biased but well-reported trial can receive a high score of quality. Inversely, a well-conducted but weakly reported trial can receive a low score of quality (2).

Assessing the quality of RCTs is a relatively important development and is usually performed via three tools of component, checklist, and scale. The component tool evaluates some aspects of a trial, whereas checklists and scales involve lists of items for the assessment of its quality. Scales provide a numeric score of quality which can be formally included into a systematic review study (2).

Meta-analyses of RCTs are being published increasingly $(2,3)$; there is, therefore, great interest in the quality assessment of the RCTs in such analyses (4-9). If safety and efficacy results of a meta-analysis are significantly affected by the quality of the original trials, then its results may be less meaningful if quality is not assessed formally (10).

There has also been a rise in the number of journals publishing RCTs. The past decade (i.e. 2000 - 2014) has witnessed an increase in the number of Iranian RCTs published in PubMed journals. Searching PubMed using the "randomized controlled trial" keyword in the [Title/Abstract] reveals an increase over time, i.e. from 15 
records between 2000 and 2005 to 92 records between 2006 and 2010 and 373 records between 2011 and 2014. Consequently, it is important for the authors, reviewers, and editors of journals to pay special attention to reporting quality assessment.

\subsection{Nephrology Urology Monthly Journal}

Nephrology Urology Monthly (NUM) is a clinical openaccess Iranian journal indexed in PubMed with an average number of published articles per year of 63. NUM is an authoritative clinical source devoted to selected compilations of the latest worldwide and interdisciplinary research and reviews in the field of basic and clinical urology and nephrology. The journal's main focus is on the efficacy in improving clinically relevant outcomes such as mortality, morbidity, and quality of life. NUM accepts all kinds of manuscripts and other scientific communications, including original manuscripts, meta-analyses and reviews, health economic papers, debates, and consensus statements of clinical relevance of nephrological and urological fields. The impact factor and rejection rate of this journal in 2012 were 0.3 and 32\%, respectively.

\subsection{Objectives}

Our primary focus was to evaluate the reporting quality of the RCTs published in NUM. As a secondary aim, we examined whether there was a change over time in the reporting quality.

\section{Evidence Acquisition}

This study is a journal-based assessment. The inclusion criterion was trials on humans with control groups published from 2012 to 2014 in NUM. We extracted descriptive information such as the year and location of the study, number and gender of patients, condition under investigation (diseases), outcomes, intervention and comparison groups, and ethical approval.

We also completed a comprehensive quality assessment of each report using the Consolidated Standards of Reporting Trials (CONSORT) and Jadad methods. The CONSORT, first conducted in 1996, comprises randomization, allocation concealment, sample size, statistical analysis, blinding, and primary and secondary outcomes (1). The objective of the CONSORT is to provide guidance to authors and reviewers about how to improve the quality of reporting. The CONSORT has been revised and published as the CONSORT 2010 statement checklist. This checklist contains multiple modified items listed separately (25 items and 37 sub-items) about title, abstract, introduction, methods, results, discussion, and additional information. Each item is reported as "yes" if the author has reported it. The CONSORT 2010 Statement and its website (www.consort-statement.org) are helpful in enhancing the reporting quality of RCTs. In the present assessment, we employed only 7 important items of the 37 sub-items of the CONSORT 2010 Statement: 1a) title and abstract: identification as a randomized trial in the title; $4 \mathrm{a}$ ) methods (participants): eligibility criteria for the participants; $4 \mathrm{~b}$ ) methods (participants): Settings and Locations where the data were collected; 5) methods (interventions): interventions for each group with sufficient details to allow replication, including how and when they were actually administered; 6a) methods (outcomes): completely defined pre-specified primary and secondary outcome measures, including how and when they were assessed; 17a) results (outcomes and estimation): for each primary and secondary outcome, results for each group, and the estimated effect size and its precision (such as 95\% confidence interval); and 23) Registration Number and the name of the trial registry.

The Jadad scale, comprised of 5 items of randomization, method of randomization, blinding, method of blinding, and dropouts and withdrawals, is used to assess quality. Each question contains a "yes" or "no" response option. In total, 5 points can be awarded, with higher points indicating superior quality. Although this scale is conducted primarily to assess the quality of the reports on pain studies, it has been used in other areas as well (8).

In the present study, two reviewers conducted all the assessments. We performed a prior training to evaluate the quality of the RCTs via the two methods. We compared the number of the checklist criteria (7 items of 37 sub-items) that were reported appropriately as specified in the CONSORT 2010 Statement checklist. We also assessed separately the items and the total quality score obtained from the Jadad scale. The mean of the number of appropriate reported items according to the CONSORT 2010 Statement checklist and the mean Jadad score over time were compared.

\section{Results}

Our database searching on PubMed identified 188 published articles from 2012 to 2014 in NUM. Of these, 170 nontrial or non-human trials were excluded and 22 trials remained. Four reports had no control group and failed to meet our eligibility criteria. Finally, 18 RCTs were selected (Figure 1). The descriptive and quality assessment information on the RCTs is depicted in Tables 1 and 2.

Fifteen (83\%) reports were submitted from Iran. In 11 (61\%) studies, the first or the corresponding author was 
Table 1. Quality Assessment via the Jadad Score for the Randomized Controlled Trials Published in Nephrology Urology Monthly From 2012 to $2014{ }^{\text {a }}$

\begin{tabular}{|c|c|c|c|c|c|c|}
\hline \multirow{2}{*}{ Reference } & \multicolumn{2}{|c|}{ Randomization } & \multicolumn{2}{|r|}{ Blinding } & \multirow{2}{*}{ Dropouts/Withdrawals } & \multirow{2}{*}{ Total Score } \\
\hline & Randomized & $\begin{array}{c}\text { Method of } \\
\text { Randomization }\end{array}$ & Blinding & Method of Blinding & & \\
\hline (11) & $0, N R$ & $0, \mathrm{NR}$ & $0, \mathrm{NR}$ & $0, \mathrm{NR}$ & 1 & 1 \\
\hline (12) & 1 & $0, N R$ & $0, N R$ & $0, N R$ & $0, \mathrm{NA}$ & 1 \\
\hline (13) & 1 & $0, N R$ & 1 & 1 & 1 & 4 \\
\hline (14) & 1 & 1 & $0, N R$ & $0, N R$ & 1 & 3 \\
\hline (15) & 1 & $0, N R$ & $0, N R$ & $0, N R$ & $0, \mathrm{NA}$ & 1 \\
\hline$(16)^{b}$ & 1 & $0, \mathrm{NR}$ & 1 & 1 & $0, \mathrm{NA}$ & 3 \\
\hline (17) & 1 & $0, N R$ & $0, N R$ & $0, N R$ & 1 & 2 \\
\hline$(18)^{\mathbf{b}}$ & 1 & $0, N R$ & 1 & 1 & 1 & 3 \\
\hline (19) & 1 & $0, N R$ & 1 & $0, N R$ & $0, \mathrm{NA}$ & 2 \\
\hline (20) & 1 & $0, N R$ & 1 & 1 & 1 & 4 \\
\hline (21) & 1 & $0, \mathrm{NR}$ & $0, N R$ & $0, N R$ & 1 & 2 \\
\hline (22) & 1 & $0, N R$ & $0, N R$ & $0, N R$ & 1 & 2 \\
\hline (23) & 1 & $0, \mathrm{NR}$ & $0, N R$ & $0, N R$ & 1 & 2 \\
\hline$(24)^{\mathbf{b}}$ & 1 & 1 & 1 & 1 & 1 & 5 \\
\hline$(25)^{\mathbf{b}}$ & 1 & $0, N R$ & $0, N R$ & $0, \mathrm{NR}$ & 1 & 2 \\
\hline (26) & 1 & $0, \mathrm{NR}$ & 1 & 1 & 1 & 5 \\
\hline (27) & 1 & 1 & 1 & 1 & 1 & 5 \\
\hline (27) & 1 & $0, N R$ & $0, \mathrm{NR}$ & $0, \mathrm{NR}$ & 1 & 2 \\
\hline
\end{tabular}

${ }^{a}$ Abbreviations: NA: not appropriate; and NR, not reported.

${ }^{\mathrm{b}}$ First author or corresponding author was affiliated to Baqiyatallah university of medical sciences.

affiliated to Baqiyatallah university of medical sciences. There were 12 (66\%) reports involving both male and female genders. In 7 (38.8\%) reports, hemodialysis and renal transplantation patients were the target group. The measured outcome in $6(33 \%)$ reports was lab data, and the intervention methods were surgery and drug therapy in 7 (38.8\%) and 10 (55.5\%) reports, respectively (Table 3 ).

The items of 1a, 4a, 4b, 5, 6a, and $17 \mathrm{a}$ and 23 items of the CONSORT 2010 Statement checklist were reported in $61.1 \%$, $94.4 \%, 66.6 \%, 94.4 \%, 83.3 \%, 88.8 \%$, and $27.7 \%$ of the RCTs, correspondingly (Table 3 ). Accordingly, the best reports were related to the eligibility criteria for the participants and the pre-specified primary and secondary outcome measures. We defined the number of the appropriate reported items as a total score for this checklist. Three studies obtained full points (7 items). The quality of reporting (by CONSORT 2010 Statement) was 4.4, 5.4, and 6 in the years 2012, 2013, and 2014, respectively, showing a trend of improvement over time. The quality of reporting in the RCTs performed by Baqiyatallah university of medical sciences, other Iranian universities, and other countries was 3.25, 3, and 1.2, respectively. Thus, the highest reporting quality in the RCTs was achieved by Baqiyatallah university of medical sciences (Table 4).

The mean score of the Jadad scale was $2.72 \pm 1.36$ (54\% of the maximum possible total score). The items of randomization, method of randomization, blinding, method of blinding, and dropouts/withdrawals of the Jadad scale were reported in $94.4 \%, 16.6 \%, 44.4 \%, 38.8 \%$, and $77.7 \%$ of the RCTs, respectively. This score was $2.7,2.8$, and 3.5 in the years 2012, 2013, and 2014, respectively, which shows an increase in the quality of reporting over time (Table 4).

\section{Discussion}

Some potential biases in RCTs may never be minimized. For example, double blinding is questionable ethically and scientifically during surgical trials (3). One way to improve the reporting quality of RCTs is to use the CONSORT 2010 Statement. The journals that adhere to this checklist in their publication of RCTs have higher reporting quality than the ones that do not (8). 


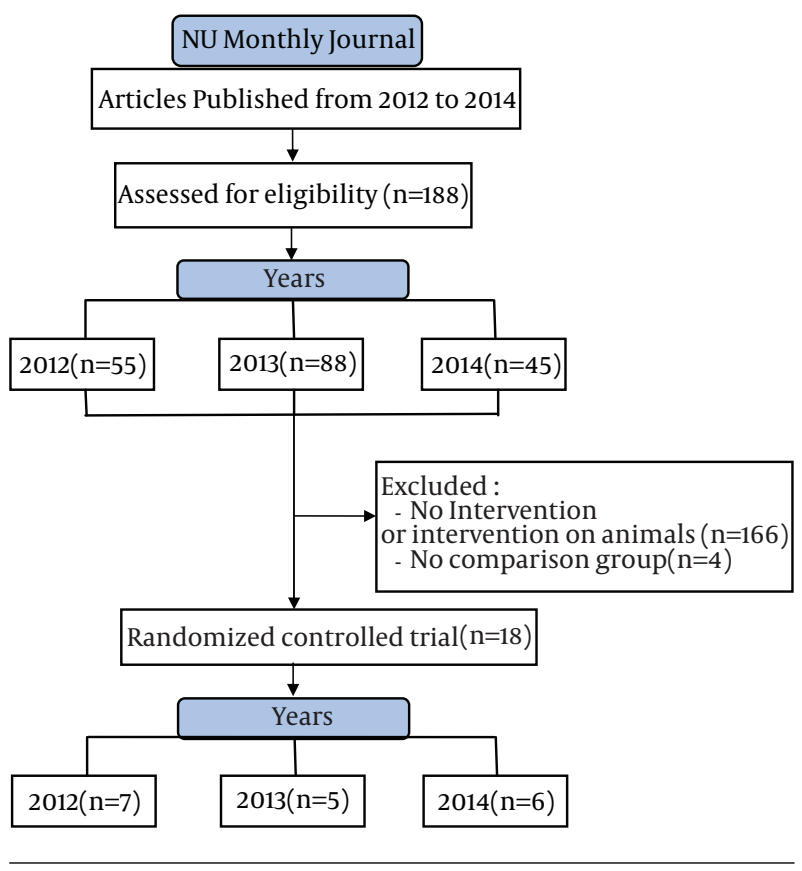

Figure 1. Tracking and Enrollment of the Randomized Controlled Trials

Proper reporting is required to generate unbiased comparison groups in controlled trials. Nonetheless, the reports of Iranian researchers in some journals usually provide inadequate or unacceptable information on critical points. Indeed, there are weaknesses in the reporting quality of the RCTs published by Iranian researchers in both Persian and English languages. A previous research suggested, however, that the quality of the non-English RCTs was similar to that in the English ones (9).

In 2012, (29), utilizing the CONSORT 2010 Statement, assessed 314 RCTs indexed in PubMed with affiliation to Tehran university of medical sciences and Iran university of medical sciences and showed that only the intervention used in the two groups was presented completely (100\%) in the abstracts. In our assessment, the best report was related to defining eligibility criteria for the participants and defining intervention for each group. The other items in the Amanollahi et al. (29) study regarding the method of randomization, method of blinding, identification as a randomized trial in the title (item 1a), eligibility criteria for the participants (item 4a), and settings and locations where the data were collected (item $4 \mathrm{~b}$ ) were reported weakly and seen in $5.4 \%, 50.3 \%, 37.6 \%, 66.4 \%$, and $19.4 \%$ of the reports, respectively. These percentages are comparable to those in our assessment. We showed that the Method of Randomization (16.6\%), Eligibility criteria (94.4\%), and Set- tings and Locations where the data were collected (66.6\%) were reported with higher quality in the RCTs included in our study.

In 2013, Ghujazadeh et al. (30), drawing on the CONSORT 2010 Statement, assessed the reporting quality of 141 RCTs published by Iranian researchers in obstetrics and gynecology level-1 journals and showed that the weaknesses were chiefly in the Methods and Material, where out of 17 items, sample size determination, method of randomization, details of any kind of randomization (e.g. categorization and block formation), and blinding method accounted for the most notable shortcomings. The authors found that the method of randomization and method of blinding were reported in $39 \%$ and $50.4 \%$ of the RCTs; these percentages are higher than those in the present study. In 2014, Faizi et al. (31) in the quality assessment of the RCTs on applied psychotherapy for chronic pains in Iran showed that the mean score of Jadad was $1.53 \pm 1.37$, while this score had a higher mean in our assessment. The authors reported that the items ( 5 items) of the Jadad score were appropriately reported in $41.2 \%, 64.7 \%, 11.8 \%, 5.9 \%$, and $29.4 \%$ of the RCTs, respectively, while these percentages in our study were $94.4 \%, 16.6 \%, 44.4 \%, 38.8 \%$, and $77.7 \%$ of the RCTs, correspondingly, which indicates a higher quality of reports in our assessment with the exception of the method of randomization item.

Moher et al. (3) evaluated the reporting quality of RCTs on pediatric alternative medicine and showed that the studies achieved approximately $40 \%$ of their maximum possible total Jadad score. This result in our study was 54.4\%.

According to the SCImago Journal Rank (SJR) indicator (http://www.scimagojr.com/journalsearch.php?q=19700201343\&tip=sid\& our findings demonstrated that the rank of NUM in 2013 was higher than that in $2012($ SJR2012 $=0.118$ vs. SJR2013 = 0.126 ). This shows that the reporting quality score can be considered a supplement to the ranking indices.

In general, meticulous reporting is required to generate an unbiased comparison of groups in RCTs. Our reporting quality assessment of the RCTs published in NUM, however, revealed low quality scores. It is possible that the Iranian investigators in this field have conducted few RCTs and therefore, not very experienced. Training courses for researchers, utilizing necessary reporting standard tools such as the CONSORT 2010 Statement by the editors of medical journals, and consultation with methodologists can improve the quality of RCTs.

\section{Acknowledgments}

We gratefully acknowledge all the individuals who helped us in this research, especially Professor Einollahi for 
his kindly collaboration.

\section{Footnote}

Authors' Contributions: Alireza Mehrazmay and Alireza Karambakhsh contributed to data extraction, analysis, and interpretation. Mahmood Salesi contributed to the critical revision and final approval of the study.

\section{References}

1. Begg C, Cho M, Eastwood S, Horton R, Moher D, Olkin I, et al. Improving the quality of reporting of randomized controlled trials. The CONSORT statement. JAMA. 1996;276(8):637-9. [PubMed: 8773637].

2. Moher D, Jadad AR, Tugwell P. Assessing the quality of randomized controlled trials. Current issues and future directions. Int J Technol Assess Health Care. 1996;12(2):195-208. [PubMed: 8707495].

3. Moher D, Soeken K, Sampson M, Ben-Porat L, Berman B. Assessing the quality of reports of systematic reviews in pediatric complementary and alternative medicine. BMC Pediatr. 2002;2:3. [PubMed: 11914146].

4. Schulz KF, Chalmers I, Grimes DA, Altman DG. Assessing the quality of randomization from reports of controlled trials published in obstetrics and gynecology journals. JAMA. 1994;272(2):125-8. [PubMed: 8015122].

5. Schulz KF, Chalmers I, Hayes RJ, Altman DG. Empirical evidence of bias. Dimensions of methodological quality associated with estimates of treatment effects in controlled trials. JAMA. 1995;273(5):40812. [PubMed: 7823387].

6. Moher D, Pham B, Jones A, Cook DJ, Jadad AR, Moher M, et al. Does quality of reports of randomised trials affect estimates of intervention efficacy reported in meta-analyses?. Lancet. 1998;352(9128):609-13. doi: 10.1016/S0140-6736(98)01085-X. [PubMed: 9746022].

7. Kjaergard LL, Villumsen J, Gluud C. Reported methodologic quality and discrepancies between large and small randomized trials in meta-analyses. Ann Intern Med. 2001;135(11):982-9. [PubMed: 11730399].

8. Jadad AR, Moore RA, Carroll D, Jenkinson C, Reynolds DJ, Gavaghan DJ, et al. Assessing the quality of reports of randomized clinical trials: is blinding necessary?. Control Clin Trials. 1996;17(1):1-12. [PubMed 8721797].

9. Moher D, Fortin P, Jadad AR, Juni P, Klassen T, Le Lorier J, et al. Completeness of reporting of trials published in languages other than English: implications for conduct and reporting of systematic reviews. Lancet. 1996;347(8998):363-6. [PubMed: 8598702].

10. Moher D, Jadad AR, Nichol G, Penman M, Tugwell P, Walsh S. Assessing the quality of randomized controlled trials: an annotated bibliography of scales and checklists. Control Clin Trials. 1995;16(1):62-73. [PubMed: 7743790].

11. Abdelrahman SS, Eassa BI. Outcome of Loupe-Assisted Sub-inguinal Varicocelectomy in Infertile Men. Nephrourol Mon. 2012;4(3):535-40. doi: 10.5812/numonthly.1623. [PubMed: 23573481]

12. Griwan MS, Kumar A, Sen J, Singh SK. Comparative evaluation of periprostatic nerve block and diclofenac patch in transrectal ultrasound-guided prostatic needle biopsy. Nephrourol Mon 2012;4(3):560-4. doi: 10.5812/numonthly.4015. [PubMed: 23573486].

13. Jalalzadeh M, Nazarian M, Vafaeimanesh J, Mirzamohammadi F. Comparison of azithromycin and clarithromycin triple therapy regimens for helicobacter pylori eradication in hemodialysis patients. Nephrourol Mon. 2012;4(3):571-7. doi: 10.5812/numonthly.2794. [PubMed: 23573488].

14. Khoshrang H, Falahatkar S, Ilat S, Akbar MH, Shakiba M, Farzan A, et al. Comparative study of hemodynamics electrolyte and metabolic changes during prone and complete supine percutaneous nephrolithotomy. Nephrourol Mon. 2012;4(4):622-8. doi: 10.5812/numonthly.4099. [PubMed: 23573503]

15. Rabani SM, Moosavizadeh A. Management of Large Proximal Ureteral Stones: A Comparative Clinical Trial Between Transureteral Lithotripsy (TUL) and Shock Wave Lithotripsy (SWL). Nephrourol Mon. 2012;4(3):556-9. doi: 10.5812/numonthly.3936. [PubMed: 23573485].

16. Rostami Z, Einollahi B, Einollahi MJ, Lessan S. The impact of amlodipine on gingival enlargement after kidney transplantation. Nephrourol Mon. 2012;4(3):565-70. doi: 10.5812/numonthly.5427. [PubMed: 23573487].

17. Sobhani Z, Ahmadi F, Jalili M, Nadia Hatmi Z, Olang O, Eslami K, et al. Comparison of two methods of teaching hypertension in under graduate medical students: "planned lecture" versus "cooperative learning". Nephrourol Mon. 2012;4(2):478-81. doi: 10.5812/numonthly.4103. [PubMed: 23573471].

18. Biniaz V, Nemati E, Tayebi A, Sadeghi Shermeh M, Ebadi A. The effect of vitamin $C$ on parathyroid hormone in patients on hemodialysis with secondary hyperparathyroidism: a double blind, placebo-controlled study. Nephrourol Mon. 2013;5(5):962-6. doi:10.5812/numonthly.12404. [PubMed: 24693502].

19. Ketabchi AA, Ketabchi M, Barkam M. The Effect of Modified TURP (M-TURP) in Intra and Postoperative Complications. Nephrourol Mon. 2013;5(2):758-61. doi: 10.5812/numonthly.6607. [PubMed: 23841040].

20. Mozafar M, Samsami M, Sobhiyeh MR, Jabbehdari S, Fallah Zavareh M. Effectiveness of aspirin on double lumen permanent catheter efficacy in ESRD. Nephrourol Mon. 2013;5(2):762-5. doi: 10.5812/numonthly.8733. [PubMed: 23841041].

21. Mozaffar M, Fallah M, Lotfollahzadeh S, Sobhiyeh MR, Gholizadeh B, Jabbehdari S, et al. Comparison of efficacy of side to side versus end to side arteriovenous fistulae formation in chronic renal failure as a permanent hemodialysis access. Nephrourol Mon. 2013;5(3):827-30. doi: 10.5812/numonthly.10248. [PubMed: 24282794].

22. Sedighi O, Makhlough A, Janbabai G, Neemi M. Comparative study of intravenous iron versus intravenous ascorbic Acid for treatment of functional iron deficiency in patients under hemodialysis: a randomized clinical trial. Nephrourol Mon. 2013;5(4):913-7. doi: 10.5812/numonthly.12038. [PubMed: 24350091].

23. Azarfar A, Esmaeeili M, Farrokh A, Alamdaran A, Keykhosravi A, Neamatshahi $\mathrm{M}$, et al. Oral midazolam for voiding dysfunction in children undergoing voiding cystourethrography: a controlled randomized clinical trial. Nephrourol Mon. 2014;6(3):eee17168. doi:10.5812/numonthly.17168. [PubMed: 25032141].

24. Biniaz V, Sadeghi Shermeh M, Ebadi A, Tayebi A, Einollahi B. Effect of Vitamin C Supplementation on C-reactive Protein Levels in Patients Undergoing Hemodialysis: A Randomized, Double Blind, PlaceboControlled Study. Nephrourol Mon. 2014;6(1):eee13351. doi: 10.5812/numonthly.13351. [PubMed: 24719806].

25. Heidari F, Abbaszadeh S, Ghadian A, Tehrani Kia F. On demand urethral dilatation versus intermittent urethral dilatation: results and complications in women with urethral stricture. Nephrourol Mon. 2014;6(2):eee15212. doi: 10.5812/numonthly.15212. [PubMed: 24783171].

26. Ketabchi AA, Mehrabi S. The effect of tamsulosin, an alpha-1 receptor antagonist as a medical expelling agent in success rate of ureteroscopic lithotripsy. Nephrourol Mon. 2014;6(1):eee12836. doi:10.5812/numonthly.12836. [PubMed: 24719805]

27. Makhlough A, Kashi Z, Akha O, Zaboli E, Yazdanicharati J. Effect of spironolactone on diabetic nephropathy compared to the combination of spironolactone and losartan. Nephrourol Mon. 2014;6(1):eee12148. doi: 10.5812/numonthly.12148. [PubMed: 24719811].

28. Mustafar R, Mohd R, Ahmad Miswan N, Cader R, Gafor HA, Mohamad $M$, et al. The effect of calcium with or without calcitriol supplementation on renal function in patients with hypovitaminosis $d$ and chronic kidney disease. Nephrourol Mon. 2014;6(1):eee13381. doi: 10.5812/numonthly.13381. [PubMed: 24719814]. 
29. Amanollahi A, Shokraneh F, Mohammadhassanzadeh H, EbrahimiKalan M, Banani G. Quality Assessment of Randomized Controlled Clinical Trials Indexed in PubMed Using CONSORT Statement [in Persian]. Health Inform Manag. 2012;44(14):7-10.

30. Ghujazadeh M. Quality of randomized clinical trial reports published by iranian researchers in the obstetrics and gynecology level 1 jour- nals: Using consort. IJOGI. 2013;16(78):7-15.

31. Faizi F, Tavallaee A, Rahimi A, Saburi A, Saghafinia M. Quality assessment of randomized control trials applied psychotherapy for chronic pains in iran: a systematic review of domestic trials. Iran Red Crescent Med J. 2014;16(9):eee15312. doi: 10.5812/ircmj.15312. [PubMed: 25593723]. 
Table 2. Randomized Controlled Trials Published in Nephrology Urology Monthly From 2012 to 2014 a

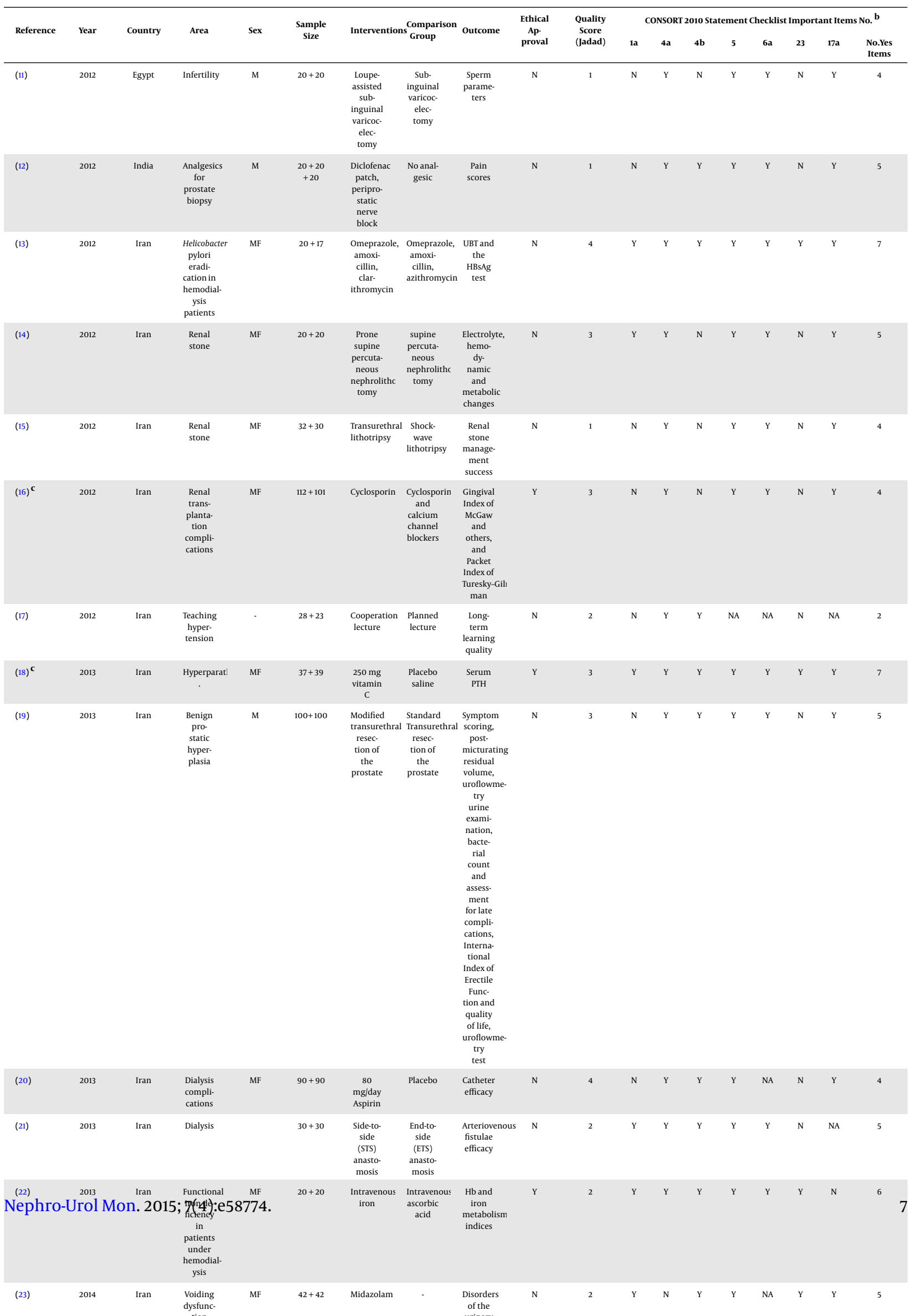


Nephro-Urol Mon. 2015; 7(4):e58774. 
Table 4. Randomized Controlled Trials Published in Nephrology Urology Monthly According to the Year of Publication

\begin{tabular}{|c|c|c|c|c|c|c|c|}
\hline & \multicolumn{3}{|c|}{ Year } & \multirow[b]{2}{*}{ Total } & \multicolumn{3}{|c|}{ Country } \\
\hline & 2012 & 2013 & 2014 & & $\operatorname{Iran}^{a}$ & Iran & Other \\
\hline $\mathbf{n}$ & 7 & 5 & 6 & 18 & & & \\
\hline Jadad scale $^{\text {b }}$ & 2.7 & 2.8 & 3.5 & 2.72 & 3.25 & 3 & 1.3 \\
\hline CONSORT 2010 Statement c & 4.42 & 5.4 & 6 & 5.27 & 5.75 & 5.09 & 5 \\
\hline
\end{tabular}

${ }^{\text {a }}$ First or corresponding author was affiliated to Baqiyatallah university of medical science.

${ }^{\mathrm{b}}$ Mean of score.

${ }^{\mathrm{c}}$ Mean of the number of appropriate reported items. 
